\title{
A targeted metabolomic approach to assess the reproducibility of plasma metabolites over a four-month period
}

\author{
X. Yin ${ }^{1}$, O. Prendiville ${ }^{1}$, A. McNamara ${ }^{1}$ and L. Brennan ${ }^{1}$ \\ ${ }^{1}$ Institute of Food and Health, UCD Conway Institute, School of Agriculture and Food Science, UCD, Belfield, Dublin, \\ Ireland
}

Metabolomics has been increasingly used in the field of nutrition research to investigate diet-disease relationships in blood or urine samples $^{(1,2)}$. However, lack of reproducibility of metabolite levels could limit their use in large-scale epidemiological studies. The present study aims to evaluate the reproducibility of metabolites over a four-month period in a free-living population.

Fasting plasma samples and dietary data from A-diet Confirm study were collected once a month, for four consecutive months. Plasma metabolites were quantified by mass spectrometry including amino acids, biogenic amines, acylcarnitines, lysophosphatidylcholines (LPCs), phosphatidylcholines (PCs), sphingomyelins (SMs), and hexoses. Metabolite reproducibility was assessed using intraclass correlation coefficient (ICC) and hierarchical clustering analysis. Diet-metabolite associations were examined by regularized canonical correlation analysis (rCCA).

A total of 148 metabolites were quantified including 21 amino acids, 12 biogenic amines, 17 acylcarnitines, 11 LPCs, 72 PCs, 14 SMs lipids, and the sum of hexoses in plasma. The median ICC for amino acids and biogenic amines was 0.76 and 0.88 , respectively. Lipid reproducibility was the highest for acylcarnitines (median ICC $=0.91$ ), and then for LPCs (median ICC $=0.88$ ), PCs (median ICC $=0.88$ ), and SMs (median ICC $=0.87$ ). The ICC for the sum of hexoses was 0.70 . All metabolites showed ICC $>0.4$ and $79.73 \%$ of metabolites were found ICC values $>0.75$. The median ICC for all metabolites was 0.88 . Additionally, the potential factors influencing lipid profiles were investigated and the rCCA indicated positive correlations between certain lipids and meat intake, dairy intake and butter/fat spread intake.

The majority of plasma metabolites demonstrated good to excellent reproducibility within individuals over a four-month period indicating that a single measurement could appropriately reflect their long-term concentration levels and may be sufficient for risk assessment in prospective epidemiological studies.

\section{References}

1. Guertin KA, Moore SC, Sampson JN, et al. (2014) Am J Clin Nutr 100, 208-217.

2. Guasch-Ferré M, Hernández-Alonso P, Drouin-Chartier JP, et al. (2021) J Nutr 151, 303-311. 